\title{
Existence of solutions for fractional multi-point boundary value problems at resonance with three-dimensional kernels
}

Wei Zhang and Wenbin Liu*

"Correspondence: wblium@163.com School of Mathematics, China University of Mining and Technology, Xuzhou, 221116, P.R. China

\section{每 Springer}

\begin{abstract}
In this paper, by using Mawhin's continuation theorem, we investigate the existence of solutions for a class of fractional differential equations with multi-point boundary value problems at resonance, and the dimension of the kernel for a fractional differential operator is three. An example is given to show our main result.
\end{abstract}

MSC: 34A08; 34B15

Keywords: fractional differential equation; multi-point boundary value problem; resonance; Mawhin's continuation theorem

\section{Introduction}

Fractional calculus as a generalization of integer-order calculus has been studied for more than 300 years. Concerning the history and basic results on fractional calculus theory, we refer to [1-5]. Fractional differential equations as an application of fractional calculus have recently been viewed as a useful mathematical model applied in numerous fields of science and engineering (see [2-11]). For example, SIS epidemic model can be described by two fractional-order differential equations of the form

$$
\left\{\begin{array}{l}
D^{\alpha_{1}} S(t)=\Lambda-\beta S I-\mu S+\phi I, \\
D^{\alpha_{1}} I(t)=\beta S I-(\phi+\mu+\alpha) I
\end{array}\right.
$$

where $D^{\alpha_{1}}$ is Caputo fractional derivatives with $0<\alpha_{1} \leq 1, S(t)$ is the number of individuals in the susceptible class at time $t$ and $I(t)$ is the number of individuals who are infectious at time $t$ (see [7]).

In recent years, boundary value problems (BVPs) and initial value problems (IVPs) of fractional differential equations have been discussed widely, and numerous valuable results have been obtained (see [12-30]). And the usual way to investigate the fractional BVPs and IVPs is nonlinear analysis such as variation method (see [12-14]), fixed-point theorems (see [15-18]), upper and lower solutions method (see [19, 20]), coincidence degree theory (see [21-25]). Besides, for the recent advances in other techniques for solving nonlinear problems, see [26-30]. For example, De La Sen et al. [29] studied the following

(c) The Author(s) 2018. This article is distributed under the terms of the Creative Commons Attribution 4.0 International License (http://creativecommons.org/licenses/by/4.0/), which permits unrestricted use, distribution, and reproduction in any medium, provided you give appropriate credit to the original author(s) and the source, provide a link to the Creative Commons license, and indicate if changes were made. 
fractional boundary value problems:

$$
\begin{aligned}
& { }^{C} D^{\alpha_{1}} x_{1}(t)=f_{1}\left(t, x_{1}(t), x_{2}(t), \ldots, x_{k}(t), I^{\beta_{11}} x_{1}(t), I^{\beta_{12}} x_{2}(t), \ldots, I^{\beta_{1 k}} x_{k}(t)\right), \\
& { }^{C} D^{\alpha_{2}} x_{2}(t)=f_{1}\left(t, x_{1}(t), x_{2}(t), \ldots, x_{k}(t), I^{\beta_{21}} x_{1}(t), I^{\beta_{22}} x_{2}(t), \ldots, I^{\beta_{2 k}} x_{k}(t)\right), \\
& \vdots \\
& { }^{C} D^{\alpha_{k}} x_{k}(t)=f_{1}\left(t, x_{1}(t), x_{2}(t), \ldots, x_{k}(t), I^{\beta_{k 1}} x_{1}(t), I^{\beta_{k 2}} x_{2}(t), \ldots, I^{\beta_{k k}} x_{k}(t)\right),
\end{aligned}
$$

which are associated with the boundary conditions

$$
x_{i}(0)+x_{i}(1)=a_{i}, \quad \sum_{j=1}^{k} I^{\beta_{i j}} x_{i}\left(\xi_{j}\right)+\sum_{j=1}^{k} I^{\beta_{i j}} x_{i}\left(\eta_{j}\right)=b_{i} \int_{0}^{1} x_{i}(s) d s,
$$

where $k$ is a natural number, $i=1,2, \ldots, k, 1 \leq \alpha_{i}<2,{ }^{C} D^{\alpha_{i}}$ denotes the Caputo fractional derivative. By using the shifted Chebyshev and Legendre polynomials approach, the authors obtained the numerical solutions for the above $k$-dimensional system.

A boundary value problem is called resonance if the corresponding homogeneous boundary value problem has a nontrivial solution. Recently, fractional boundary value problems at resonance have attracted many scholars' attention (see [21-25]). To our knowledge, the most effective method for solving fractional resonance boundary value problems is Mawhin's continuation theorem (see [31, 32]). For example, in [22], Chen and Tang considered the solvability of the following fractional-order multi-point boundary value problems at resonance by using Mawhin's continuation theorem.

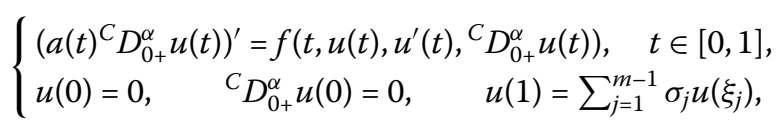

where ${ }^{C} D_{0+}^{\alpha}$ is the Caputo fractional derivative with $1<\alpha \leq 2,0<\sigma_{j} \in \mathbb{R}, \xi_{j} \in(0,1)$, $\sum_{j=1}^{m-1} \sigma_{j} \xi_{j}=1, f:[0,1] \times \mathbb{R}^{3} \rightarrow \mathbb{R}$ satisfies the Carathéodory conditions.

In [23], Bai and Zhang considered the solvability of the following fractional multipoint boundary value problems at resonance with two-dimensional kernels by employing Mawhin's continuation theorem.

$$
\left\{\begin{array}{l}
D_{0+}^{\alpha} u(t)=f\left(t, u(t), D_{0+}^{\alpha-2} u(t), D_{0+}^{\alpha-1} u(t)\right), \quad t \in(0,1), \\
I_{0+}^{3-\alpha} u(0)=0, \quad D_{0+}^{\alpha-1} u(0)=D_{0+}^{\alpha-1} u(\eta), \quad u(1)=\sum_{i=1}^{m} \alpha_{i} u\left(\eta_{i}\right),
\end{array}\right.
$$

where $D_{0+}^{\alpha}$ is the standard Riemann-Liouville fractional derivative with $2<\alpha \leq 3$, $0<\eta \leq 1,0<\eta_{1}<\cdots<\eta_{m}<1, m \geq 2, \sum_{i=1}^{m} \alpha_{i} \eta_{i}^{\alpha-1}=\sum_{i=1}^{m} \alpha_{i} \eta_{i}^{\alpha-2}=1, f:[0,+\infty) \times \mathbb{R}^{3} \rightarrow \mathbb{R}$ satisfies the Carathéodory conditions. In order to make sure that the linear operator $Q$ is well defined, the author assumed that the following condition holds:

$$
R=\frac{\eta^{\alpha} \Gamma(\alpha) \Gamma(\alpha-1)}{\alpha \Gamma(2 \alpha-1)}\left(1-\sum_{i=1}^{m} \alpha_{i} \eta_{i}^{2 \alpha-2}\right)-\frac{\eta^{\alpha-1}(\Gamma(\alpha))^{2}}{(\alpha-1) \Gamma(2 \alpha)}\left(1-\sum_{i=1}^{m} \alpha_{i} \eta_{i}^{2 \alpha-1}\right) \neq 0
$$

In [24], Jiang studied the existence of solutions for the following fractional multipoint boundary value problems at resonance with two-dimensional kernels by applying 
Mawhin's continuation theorem.

$$
\left\{\begin{array}{l}
D_{0+}^{\alpha} u(t)=f\left(t, u(t), D_{0+}^{\alpha-1} u(t)\right), \quad \text { a.e. } t \in[0,1], \\
u(0)=0, \quad D_{0+}^{\alpha-1} u(0)=\sum_{i=1}^{m} a_{i} D_{0+}^{\alpha-1} u\left(\xi_{i}\right), \quad D_{0+}^{\alpha-2} u(1)=\sum_{j=1}^{n} b_{j} D_{0+}^{\alpha-2} u\left(\eta_{j}\right),
\end{array}\right.
$$

where $D_{0_{+}}^{\alpha}$ is the standard Riemann-Liouville fractional derivative with $2<\alpha<3,0<$ $\xi_{1}<\xi_{2}<\cdots<\xi_{m}<1,0<\eta_{1}<\eta_{2}<\cdots<\eta_{n}<1, \sum_{i=1}^{m} a_{i}=\sum_{j=1}^{n} b_{j}=\sum_{j=1}^{n} b_{j} \eta_{j}=1, f:$ $[0,1] \times \mathbb{R}^{2} \rightarrow \mathbb{R}$ satisfies the Carathéodory conditions. In order to make sure that the linear operator $Q$ is well defined, the author assumed that the following condition holds:

$$
\frac{1}{6}\left(1-\sum_{j=1}^{n} b_{j} \eta_{j}^{3}\right) \sum_{i=1}^{m} a_{i} \xi_{i}-\frac{1}{4}\left(1-\sum_{j=1}^{n} b_{j} \eta_{j}^{2}\right) \sum_{i=1}^{m} a_{i} \xi_{i}^{2} \neq 0 .
$$

Thus, motivated by the results mentioned, in this paper, we discuss the existence of solutions for the following multi-point boundary value problems by using Mawhin's continuation theorem.

$$
\left\{\begin{array}{l}
D_{0+}^{\alpha} u(t)=f\left(t, u(t), D_{0+}^{\alpha-3} u(t), D_{0+}^{\alpha-2} u(t), D_{0+}^{\alpha-1} u(t)\right), \quad t \in(0,1), \\
\left.I_{0+}^{-\alpha} u(t)\right|_{t=0}=0, \quad D_{0+}^{\alpha-1} u(0)=\sum_{i=1}^{l} \alpha_{i} D_{0+}^{\alpha-1} u\left(\xi_{i}\right), \\
D_{0+}^{\alpha-2} u(0)=\sum_{j=1}^{m} \beta_{j} D_{0+}^{\alpha-2} u\left(\eta_{j}\right), \quad D_{0+}^{\alpha-3} u(1)=\sum_{k=1}^{n} \gamma_{k} D_{0+}^{\alpha-3} u\left(\rho_{k}\right),
\end{array}\right.
$$

where $D_{0+}^{\alpha}$ is the standard Riemann-Liouville fractional derivative with $3<\alpha \leq 4,0<\xi_{1}<$ $\cdots<\xi_{l}<\eta_{1}<\cdots<\eta_{m}<1,0<\rho_{1}<\cdots<\rho_{k}<1, \alpha_{i}, \beta_{j}, \gamma_{k} \in \mathbb{R}, f \in[0,1] \times \mathbb{R}^{4} \rightarrow \mathbb{R}$ is a Carathéodory function.

Throughout this paper, we assume that the following resonance conditions of (1.1) hold.

$\left(\mathrm{H}_{1}\right) \sum_{i=1}^{l} \alpha_{i}=1, \sum_{j=1}^{m} \beta_{j}=1, \sum_{k=1}^{n} \gamma_{k}=1, \sum_{j=1}^{m} \beta_{j} \eta_{j}=0, \sum_{k=1}^{n} \gamma_{k} \rho_{k}=1, \sum_{k=1}^{n} \gamma_{k} \rho_{k}^{2}=1$.

Compared with previous work in the field, in this paper several new features can be shown as follows. Firstly, to the best of author's knowledge, there are only few papers that consider the integral-order resonance BVPs with three-dimensional kernels (see [33-36]); and for fractional resonance boundary value problems, most of the discussions are limited to the kernels of operator dimension less than or equal to two. So, our results are a generalization of some previous publications. Secondly, compared with [23, 24, 35, 36], in this paper, based on analysis as proved, we needn't ensure that the linear operator $Q$ is well defined by assumed conditions. Compared with [33,34], it is more difficult to define the linear isomorphism operator $J$ and also it is even more difficult to give an example that satisfies all the assumptions in the paper. Furthermore, when we take $\alpha=4$, BVPs (1.1) are reduced to the fourth-order differential equation resonance BVPs.

We organized the rest of the article as follows. In Section 2, we recall some definitions and lemmas. In Section 3, based on Mawhin's continuation theorem, we establish an existence theorem for problem (1.1). In Section 4, we present an example to illustrate our main result. In the last section, we give a short conclusion.

\section{Preliminaries}

In this section, we recall some definitions and lemmas which are used throughout this paper. 
Let $X$ and $Y$ be two Banach spaces with the norms $\|\cdot\|_{X}$ and $\|\cdot\|_{Y}$, respectively. Define $L: \operatorname{dom}(L) \subset X \rightarrow Y$ to be a Fredholm operator with index zero, $P: X \rightarrow X, Q: Y \rightarrow Y$ to be two projectors such that

$$
\operatorname{Im} P=\operatorname{Ker} L, \quad \operatorname{Im} L=\operatorname{Ker} Q, \quad X=\operatorname{Ker} L \oplus \operatorname{Ker} P, \quad Y=\operatorname{Im} L \oplus \operatorname{Im} Q,
$$

$\left.L\right|_{\operatorname{dom} L \cap \operatorname{Ker} P}: \operatorname{dom} L \rightarrow \operatorname{Im} L$ is invertible, we denote the inverse by $K_{p}$. Let $\Omega$ be an open bounded subset of $X$ and $\operatorname{dom} L \cap \bar{\Omega} \neq \emptyset$, the map $N: X \rightarrow Y$ is called L-compact on $\bar{\Omega}$, if $Q N(\bar{\Omega})$ is bounded and $K_{P, Q} N=K_{p}(I-Q) N: \bar{\Omega} \rightarrow X$ is compact (see [31, 32]).

Lemma 2.1 (see [31, 32]) Let $L: \operatorname{dom} L \subset X \rightarrow Y$ be a Fredholm operator of index zero and $N: X \rightarrow Y$ be L-compact on $\bar{\Omega}$. Assume that the following conditions are satisfied:

(i) $L u \neq \lambda N u$ for any $u \in(\operatorname{dom} L \backslash \operatorname{Ker} L) \cap \partial \Omega, \lambda \in(0,1)$;

(ii) $N u \notin \operatorname{Im} L$ for any $u \in \operatorname{Ker} L \cap \partial \Omega$;

(iii) $\operatorname{deg}\left(\left.Q N\right|_{\operatorname{Ker} L}, \Omega \cap \operatorname{Ker} L, 0\right) \neq 0$.

Then the equation $L x=N x$ has at least one solution in $\operatorname{dom} L \cap \bar{\Omega}$.

Definition 2.1 (see $[2,3]$ ) The Riemann-Liouville fractional integral of order $\alpha>0$ for a function $u:(0,+\infty) \rightarrow \mathbb{R}$ is given by

$$
I_{0+}^{\alpha} u(t)=\frac{1}{\Gamma(\alpha)} \int_{0}^{t}(t-s)^{\alpha-1} u(s) d s
$$

provided that the right-hand side integral is pointwise defined on $(0,+\infty)$.

Definition 2.2 (see [2,3]) The Riemann-Liouville fractional derivative of order $\alpha>0$ for a function $u:(0,+\infty) \rightarrow \mathbb{R}$ is given by

$$
D_{0+}^{\alpha} u(t)=\frac{d^{n}}{d t^{n}} I_{0+}^{n-\alpha} u(t)=\frac{1}{\Gamma(n-\alpha)} \frac{d^{n}}{d t^{n}} \int_{0}^{t}(t-s)^{n-\alpha-1} u(s) d s,
$$

where $n=[\alpha]+1$, provided that the right-hand side integral is pointwise defined on $(0,+\infty)$.

Lemma 2.2 (see $[2,3,18,24])$ Let $\alpha>0$. Assume that $u, D_{0+}^{\alpha} u \in L^{1}(0,1)$, then the following equality holds:

$$
I_{0+}^{\alpha} D_{0+}^{\alpha} u(t)=u(t)+c_{1} t^{\alpha-1}+c_{2} t^{\alpha-2}+\cdots+c_{n} t^{\alpha-n},
$$

where $n=[\alpha]+1, c_{i} \in \mathbb{R}, i=1,2, \ldots, n$.

Lemma 2.3 (see $[2,3,18,24]$ ) Assume that $u \in L^{1}(0,1), \alpha \geq \beta \geq 0$, then

$$
I_{0+}^{\alpha} I_{0+}^{\beta} u(t)=I_{0+}^{\alpha+\beta} u(t), \quad D_{0+}^{\beta} I_{0+}^{\alpha} u(t)=I_{0+}^{\alpha-\beta} u(t) .
$$

Lemma 2.4 (see $[2,3,18,24])$ Assume that $\alpha>0, \lambda>-1, t>0$, then

$$
I_{0+}^{\alpha} t^{\lambda}=\frac{\Gamma(\lambda+1)}{\Gamma(\lambda+1+\alpha)} t^{\alpha+\lambda}, \quad D_{0+}^{\alpha} t^{\lambda}=\frac{\Gamma(\lambda+1)}{\Gamma(\lambda+1-\alpha)} t^{\lambda-\alpha},
$$

in particular $D_{0+}^{\alpha} t^{\alpha-m}=0, m=1,2, \ldots, n$, where $n=[\alpha]+1$. 


\section{Main result}

Take

$$
X=\left\{u: u, D_{0+}^{\alpha-1} u \in C[0,1]\right\}, \quad Y=L^{1}[0,1] .
$$

It is easy to check that $X$ and $Y$ are two Banach spaces with norms

$$
\|u\|_{X}=\max \left\{\|u\|_{\infty},\left\|D_{0+}^{\alpha-3} u\right\|_{\infty},\left\|D_{0+}^{\alpha-2} u\right\|_{\infty},\left\|D_{0+}^{\alpha-1} u\right\|_{\infty}\right\}, \quad\|y\|_{Y}=\|y\|_{1},
$$

respectively, where $\|u\|_{\infty}=\sup _{t \in[0,1]}|u(t)|,\|y\|_{1}=\int_{0}^{1}|y(t)| d t$.

Define the linear operator $L: \operatorname{dom} L \subset X \rightarrow Y$ and the nonlinear operator $N: X \rightarrow Y$ as follows:

$$
\begin{aligned}
& L u(t)=D_{0+}^{\alpha} u(t), \quad u(t) \in \operatorname{dom} L, \\
& N u(t)=f\left(t, u(t), D_{0+}^{\alpha-3} u(t), D_{0+}^{\alpha-2} u(t), D_{0+}^{\alpha-1} u(t)\right), \quad u(t) \in X,
\end{aligned}
$$

where

$\operatorname{dom} L=\left\{u \in X: D_{0_{+}}^{\alpha} u(t) \in Y, u\right.$ satisfies boundary value conditions of (1.1) $\}$.

Then problem (1.1) is equivalent to the operator equation $L u=N u, u \in \operatorname{dom} L$.

Lemma 3.1 Assume that $\left(\mathrm{H}_{1}\right)$ holds, then the operator $L: \operatorname{dom} L \subset X \rightarrow Y$ satisfies

$$
\begin{aligned}
& \operatorname{Ker} L=\left\{u \in \operatorname{dom} L: u(t)=a t^{\alpha-1}+b t^{\alpha-2}+c t^{\alpha-3}, a, b, c \in \mathbb{R}\right\}, \\
& \operatorname{Im} L=\left\{y \in Y: Q_{1} y=Q_{2} y=Q_{3} y=0\right\}
\end{aligned}
$$

where

$$
\begin{aligned}
& Q_{1} y=\sum_{i=1}^{l} \alpha_{i} \int_{0}^{\xi_{i}} y(s) d s, \quad Q_{2} y=\sum_{j=1}^{m} \beta_{j} \int_{0}^{\eta_{j}}\left(\eta_{j}-s\right) y(s) d s, \\
& Q_{3} y=\int_{0}^{1}(1-s)^{2} y(s) d s-\sum_{k=1}^{n} \gamma_{k} \int_{0}^{\rho_{k}}\left(\rho_{k}-s\right)^{2} y(s) d s .
\end{aligned}
$$

Proof If $L u=D_{0+}^{\alpha} u=0$, by Lemma 2.2, we have

$$
u(t)=a t^{\alpha-1}+b t^{\alpha-2}+c t^{\alpha-3}+d t^{\alpha-4}, \quad a, b, c, d \in \mathbb{R} .
$$

It follows from the boundary condition $\left.I_{0+}^{4-\alpha} u(t)\right|_{t=0}=0$ that $d=0$, then

$$
u(t)=a t^{\alpha-1}+b t^{\alpha-2}+c t^{\alpha-3} .
$$

So,

$\operatorname{Ker} L \subset\left\{u \in \operatorname{dom} L: u(t)=a t^{\alpha-1}+b t^{\alpha-2}+c t^{\alpha-3}, a, b, c \in \mathbb{R}\right\}$. 
Conversely, take $u(t)=t^{\alpha-1}+t^{\alpha-2}+t^{\alpha-3}$, it is easy to check that $D_{0+}^{\alpha} u=0$ and $u(t)$ satisfies boundary value conditions of (1.1). Thus,

$$
\left\{u \in \operatorname{dom} L: u(t)=a t^{\alpha-1}+b t^{\alpha-2}+c t^{\alpha-3}, a, b, c \in \mathbb{R}\right\} \subset \operatorname{Ker} L .
$$

For $y \in \operatorname{Im} L$, there exists $u \in \operatorname{dom} L$ such that $D_{0_{+}}^{\alpha} u(t)=y(t)$. By Lemmas 2.2 and 2.4, combined with the boundary condition $\left.I_{0+}^{4-\alpha} u(t)\right|_{t=0}=0$, one has

$$
u(t)=I_{0+}^{\alpha} y(t)+c_{1} t^{\alpha-1}+c_{2} t^{\alpha-2}+c_{3} t^{\alpha-3} .
$$

Considering the boundary conditions

$$
\begin{aligned}
& D_{0+}^{\alpha-1} u(0)=\sum_{i=1}^{l} \alpha_{i} D_{0+}^{\alpha-1} u\left(\xi_{i}\right), \quad D_{0+}^{\alpha-2} u(0)=\sum_{j=1}^{m} \beta_{j} D_{0+}^{\alpha-2} u\left(\eta_{j}\right), \\
& D_{0+}^{\alpha-3} u(1)=\sum_{k=1}^{n} \gamma_{k} D_{0+}^{\alpha-3} u\left(\rho_{k}\right),
\end{aligned}
$$

by Lemmas 2.3 and 2.4, we obtain

$$
\begin{aligned}
D_{0+}^{\alpha-1} u(0) & =c_{1} \Gamma(\alpha)=\sum_{i=1}^{l} \alpha_{i} D_{0+}^{\alpha-1} u\left(\xi_{i}\right) \\
& =\sum_{i=1}^{l} \alpha_{i}\left[\int_{0}^{\xi_{i}} y(s) d s+c_{1} \Gamma(\alpha)\right] \\
& =\sum_{i=1}^{l} \alpha_{i} \int_{0}^{\xi_{i}} y(s) d s+c_{1} \Gamma(\alpha), \\
D_{0+}^{\alpha-2} u(0) & =c_{2} \Gamma(\alpha-1)=\sum_{j=1}^{m} \beta_{j} D_{0+}^{\alpha-2} u\left(\eta_{j}\right) \\
& =\sum_{j=1}^{m} \beta_{j}\left[\int_{0}^{\eta_{j}}\left(\eta_{j}-s\right) y(s) d s+c_{1} \Gamma(\alpha) \eta_{j}+c_{2} \Gamma(\alpha-1)\right] \\
& =\sum_{j=1}^{m} \beta_{j} \int_{0}^{\eta_{j}}\left(\eta_{j}-s\right) y(s) d s+c_{2} \Gamma(\alpha-1)
\end{aligned}
$$

and

$$
\begin{aligned}
D_{0+}^{\alpha-3} u(1) & =\frac{1}{2} \int_{0}^{1}(1-s)^{2} y(s) d s+\frac{1}{2} c_{1} \Gamma(\alpha)+c_{2} \Gamma(\alpha-1)+c_{3} \Gamma(\alpha-2) \\
& =\sum_{k=1}^{n} \gamma_{k} D_{0+}^{\alpha-3} u\left(\rho_{k}\right) \\
& =\sum_{k=1}^{n} \gamma_{k}\left[\frac{1}{2} \int_{0}^{\rho_{k}}\left(\rho_{k}-s\right)^{2} y(s) d s+\frac{1}{2} c_{1} \Gamma(\alpha) \rho_{k}^{2}+c_{2} \Gamma(\alpha-1) \rho_{k}+c_{3} \Gamma(\alpha-2)\right] \\
& =\frac{1}{2} \sum_{k=1}^{n} \gamma_{k} \int_{0}^{\rho_{k}}\left(\rho_{k}-s\right)^{2} y(s) d s+\frac{1}{2} c_{1} \Gamma(\alpha)+c_{2} \Gamma(\alpha-1)+c_{3} \Gamma(\alpha-2) .
\end{aligned}
$$


Thus,

$$
Q_{1} y=Q_{2} y=Q_{3} y=0
$$

that is,

$\operatorname{Im} L \subset\left\{y \in Y: Q_{1} y=Q_{2} y=Q_{3} y=0\right\}$.

Conversely, let $y \in Y$ satisfy (3.3), taking $u(t)=I_{0+}^{\alpha} y(t)$, we can easily check that $u \in \operatorname{dom} L$ and $L u(t)=y(t)$. Then we have $\left\{y \in Y: Q_{1} y=Q_{2} y=Q_{3} y=0\right\} \subset \operatorname{Im} L$.

Let $R_{1}, R_{2}, R_{3}: Y \rightarrow Y$ be three linear operators defined as

$$
\begin{aligned}
& R_{1} y=\frac{\mu(\mu+1)(\mu+2)}{\Delta(\mu, v, \omega)}\left[A_{11} Q_{1} y+A_{12} Q_{2} y+A_{13} Q_{3} y\right], \\
& R_{2} y=\frac{\nu(v+1)(v+2)}{\Delta(\mu, \nu, \omega)}\left[A_{21} Q_{1} y+A_{22} Q_{2} y+A_{23} Q_{3} y\right], \\
& R_{3} y=\frac{\omega(\omega+1)(\omega+2)}{\Delta(\mu, v, \omega)}\left[A_{31} Q_{1} y+A_{32} Q_{2} y+A_{33} Q_{3} y\right],
\end{aligned}
$$

where

$$
\Delta(\mu, \nu, \omega)=\left|\begin{array}{ccc}
(\mu+1)(\mu+2) \sum_{i=1}^{l} \alpha_{i} \xi_{i}^{\mu} & (\mu+2) \sum_{j=1}^{m} \beta_{j} \eta_{j}^{\mu+1} & 2\left(1-\sum_{k=1}^{n} \gamma_{k} \rho_{k}^{\mu+2}\right) \\
(v+1)(v+2) \sum_{i=1}^{l} \alpha_{i} \xi_{i}^{\nu} & (v+2) \sum_{j=1}^{m} \beta_{j} \eta_{j}^{\nu+1} & 2\left(1-\sum_{k=1}^{n} \gamma_{k} \rho_{k}^{v+2}\right) \\
(\omega+1)(\omega+2) \sum_{i=1}^{l} \alpha_{i} \xi_{i}^{\omega} & (\omega+2) \sum_{j=1}^{m} \beta_{j} \eta_{j}^{\omega+1} & 2\left(1-\sum_{k=1}^{n} \gamma_{k} \rho_{k}^{\omega+2}\right)
\end{array}\right|
$$

We note

$$
\Delta(\mu, \nu, \omega):=\left|\begin{array}{lll}
a_{11} & a_{12} & a_{13} \\
a_{21} & a_{22} & a_{23} \\
a_{31} & a_{32} & a_{33}
\end{array}\right|
$$

here $A_{i j}(i, j=1,2,3)$ are the algebraic complements of $a_{i j}$.

Lemma 3.2 Assume that $\left(\mathrm{H}_{1}\right)$ holds, then there exist constants $\mu \in\{1,2, \ldots, m-1\}, v \in \mathbb{Z}^{+}$, $v \geq \mu+1$ and $\omega \in \mathbb{Z}^{+}$large enough numbers such that $\Delta(\mu, v, \omega) \neq 0$.

Proof By $\sum_{i=1}^{l} \alpha_{i}=1$, we have that, for each $s \in \mathbb{Z}^{+}$, there exists $k_{s} \in\{s l+1, \ldots,(s+1) l\}$ such that $\sum_{i=1}^{l} \alpha_{i} \xi_{i}^{k_{s}} \neq 0$. If not, we get $\sum_{i=1}^{l} \alpha_{i} \xi_{i}^{k_{s}}=0, k_{s} \in\{s l+1, \ldots,(s+1) l\}$, that is,

$$
\left(\begin{array}{cccc}
\xi_{1}^{s l+1} & \xi_{2}^{s l+1} & \ldots & \xi_{l}^{s l+1} \\
\xi_{1}^{s l+2} & \xi_{2}^{s l+2} & \ldots & \xi_{l}^{s l+2} \\
\vdots & \vdots & \ddots & \vdots \\
\xi_{1}^{(s+1) l} & \xi_{2}^{(s+1) l} & \ldots & \xi_{l}^{(s+1) l}
\end{array}\right)\left(\begin{array}{c}
\alpha_{1} \\
\alpha_{2} \\
\vdots \\
\alpha_{l}
\end{array}\right)=0
$$


Since

$$
\left|\begin{array}{cccc}
\xi_{1}^{s l+1} & \xi_{2}^{s l+1} & \ldots & \xi_{l}^{s l+1} \\
\xi_{1}^{s l+2} & \xi_{2}^{s l+2} & \cdots & \xi_{l}^{s l+2} \\
\vdots & \vdots & \ddots & \vdots \\
\xi_{1}^{(s+1) l} & \xi_{2}^{(s+1) l} & \cdots & \xi_{l}^{(s+1) l}
\end{array}\right|=\left(\xi_{1} \xi_{2} \cdots \xi_{l}\right)^{s l+1} \prod_{1 \leq i<j \leq l}\left(\xi_{j}-\xi_{i}\right) \neq 0
$$

So, $\alpha_{i}=0(i=1,2, \ldots, l)$, which is a contradiction with $\sum_{i=1}^{l} \alpha_{i}=1$. Similarly, by $\sum_{j=1}^{m} \beta_{j}=1$ and $\sum_{j=1}^{m} \beta_{j} \eta_{j}=0$, there exists $\mu \in\{1,2, \ldots, m-1\}$ such that $\sum_{j=1}^{m} \beta_{j} \eta_{j}^{\mu+1} \neq 0$. Otherwise, we have $\sum_{j=1}^{m} \beta_{j} \eta_{j}^{\mu+1}=0, \mu=0,1,2, \ldots, m-1$, that is,

$$
\left(\begin{array}{cccc}
\eta_{1} & \eta_{2} & \cdots & \eta_{m} \\
\eta_{1}^{2} & \eta_{2}^{2} & \cdots & \eta_{m}^{2} \\
\vdots & \vdots & \ddots & \vdots \\
\eta_{1}^{m} & \eta_{2}^{m} & \cdots & \eta_{m}^{m}
\end{array}\right)\left(\begin{array}{c}
\beta_{1} \\
\beta_{2} \\
\vdots \\
\beta_{m}
\end{array}\right)=0
$$

Because

$$
\left|\begin{array}{cccc}
\eta_{1} & \eta_{2} & \cdots & \eta_{m} \\
\eta_{1}^{2} & \eta_{2}^{2} & \cdots & \eta_{m}^{2} \\
\vdots & \vdots & \ddots & \vdots \\
\eta_{1}^{m} & \eta_{2}^{m} & \cdots & \eta_{m}^{m}
\end{array}\right|=\eta_{1} \eta_{2} \cdots \eta_{m} \prod_{1 \leq i<j \leq m}\left(\eta_{j}-\eta_{i}\right) \neq 0
$$

So, $\beta_{j}=0(j=1,2, \ldots, m)$, which has conflicts with $\sum_{j=1}^{m} \beta_{j}=1$. Set

$$
S=\left\{k_{s} \in \mathbb{Z}^{+}: \frac{(\mu+1) \sum_{i=1}^{l} \alpha_{i} \xi_{i}^{\mu} \sum_{j=1}^{m} \beta_{j} \eta_{j}^{k_{s}+1}}{\left(k_{s}+1\right) \sum_{i=1}^{l} \alpha_{i} \xi_{i}^{k_{s}}}=\sum_{j=1}^{m} \beta_{j} \eta_{j}^{\mu+1}\right\}
$$

We show that $S$ is a finite set. If else, there exists a strictly monotonic sequence $\left\{k_{s t}\right\}_{t=1}^{\infty}$ such that

$$
\frac{(\mu+1) \sum_{i=1}^{l} \alpha_{i} \xi_{i}^{\mu} \sum_{j=1}^{m} \beta_{j} \eta_{j}^{k_{s}+1}}{\left(k_{s_{t}}+1\right) \sum_{i=1}^{l} \alpha_{i} \xi_{i}^{k_{s t}}}=\sum_{j=1}^{m} \beta_{j} \eta_{j}^{\mu+1}
$$

It follows from $0<\xi_{1}<\cdots<\xi_{l}<\eta_{1}<\cdots<\eta_{m}<1, \sum_{j=1}^{m} \beta_{j} \eta_{j}^{\mu+1} \neq 0$ that $\sum_{i=1}^{l} \alpha_{i} \xi_{i}^{\mu} \neq 0$ and

$$
\begin{aligned}
\sum_{j=1}^{m} \beta_{j} \eta_{j}^{\mu+1} & =\lim _{k_{s} \rightarrow \infty} \frac{(\mu+1) \sum_{i=1}^{l} \alpha_{i} \xi_{i}^{\mu} \sum_{j=1}^{m} \beta_{j} \eta_{j}^{k_{s t}+1}}{\left(k_{s_{t}}+1\right) \sum_{i=1}^{l} \alpha_{i} \xi_{i}^{k_{s}}} \\
& =\lim _{k_{s t} \rightarrow \infty} \frac{\xi_{l}(\mu+1) \sum_{i=1}^{l} \alpha_{i} \xi_{i}^{\mu} \sum_{j=1}^{m} \beta_{j}\left(\eta_{j} / \xi_{l}\right)^{k_{s t}+1}}{\left(k_{s_{t}}+1\right) \sum_{i=1}^{l} \alpha_{i}\left(\xi_{i} / \xi_{l}\right)^{k_{s t}}}=\infty .
\end{aligned}
$$


It is a contradiction. Thus, there exist $\mu \in\{1,2, \ldots, m-1\}, v \in \mathbb{Z}^{+}, v \geq \mu+1$ such that $A_{33} \neq 0$. Therefore, we have

$$
\lim _{\omega \rightarrow \infty} \Delta(\mu, \nu, \omega)=\left|\begin{array}{ccc}
a_{11} & a_{12} & a_{13} \\
a_{21} & a_{22} & a_{23} \\
0 & 0 & 2
\end{array}\right|=2 A_{33} \neq 0 .
$$

So, if we make sure $\omega \in \mathbb{Z}^{+}$(the set of positive integers) is large enough, it can be such that $\Delta(\mu, \nu, \omega) \neq 0$.

Lemma 3.3 Assume that $\left(\mathrm{H}_{1}\right)$ holds, then $L: \operatorname{dom} L \subset X \rightarrow Y$ is a Fredholm operator of index zero. The linear projector operator $P: X \rightarrow X$ and $Q: Y \rightarrow Y$ can be defined as follows:

$$
\begin{aligned}
& (P u)(t)=\frac{1}{\Gamma(\alpha)} D_{0+}^{\alpha-1} u(0) t^{\alpha-1}+\frac{1}{\Gamma(\alpha-1)} D_{0+}^{\alpha-2} u(0) t^{\alpha-2}+\frac{1}{\Gamma(\alpha-2)} D_{0+}^{\alpha-3} u(0) t^{\alpha-3}, \\
& (Q y)(t)=\left(R_{1} y(t)\right) t^{\mu-1}+\left(R_{2} y(t)\right) t^{\nu-1}+\left(R_{3} y(t)\right) t^{\omega-1},
\end{aligned}
$$

where taking $\mu, \nu, \omega$ satisfies Lemma 3.2.

Proof By the definition of $P$, we can easily check that $P$ is a continuous linear projector operator and satisfies $\operatorname{Im} P=\operatorname{Ker} L, X=\operatorname{Ker} P \oplus \operatorname{Ker} L$. According to Lemma 3.2, there exist constants $\mu \in\{1,2, \ldots, m-1\}, v \in \mathbb{Z}^{+}, v \geq \mu+1$ and $\omega \in \mathbb{Z}^{+}$large enough, such that $\Delta(\mu, \nu, \omega) \neq 0$. So, $Q$ is a well-defined operator. It is clear that $Q$ is a continuous linear operator and $\operatorname{dim} \operatorname{Im} Q=3$. By the definitions of $R_{1}, R_{2}, R_{3}$, we can calculate the following equations:

$$
\begin{array}{lrrr}
R_{1}\left(\left(R_{1} y\right) t^{\mu-1}\right)=R_{1} y, & R_{1}\left(\left(R_{2} y\right) t^{\nu-1}\right)=0, & R_{1}\left(\left(R_{3} y\right) t^{\omega-1}\right)=0, \\
R_{2}\left(\left(R_{1} y\right) t^{\mu-1}\right)=0, & R_{2}\left(\left(R_{2} y\right) t^{\nu-1}\right)=R_{2} y, & R_{2}\left(\left(R_{3} y\right) t^{\omega-1}\right)=0, \\
R_{3}\left(\left(R_{1} y\right) t^{\mu-1}\right)=0, & R_{3}\left(\left(R_{2} y\right) t^{\nu-1}\right)=0, & R_{3}\left(\left(R_{3} y\right) t^{\omega-1}\right)=R_{3} y .
\end{array}
$$

Thus,

$$
\begin{aligned}
\left(Q^{2} y\right)(t)= & Q\left[\left(R_{1} y(t)\right) t^{\mu-1}+\left(R_{2} y(t)\right) t^{\nu-1}+\left(R_{3} y(t)\right) t^{\omega-1}\right] \\
= & \left\{R_{1}\left[\left(R_{1} y(t)\right) t^{\mu-1}+\left(R_{2} y(t)\right) t^{\nu-1}+\left(R_{3} y(t)\right) t^{\omega-1}\right]\right\} t^{\mu-1} \\
& +\left\{R_{2}\left[\left(R_{1} y(t)\right) t^{\mu-1}+\left(R_{2} y(t)\right) t^{\nu-1}+\left(R_{3} y(t)\right) t^{\omega-1}\right]\right\} t^{\nu-1} \\
& +\left\{R_{3}\left[\left(R_{1} y(t)\right) t^{\mu-1}+\left(R_{2} y(t)\right) t^{\nu-1}+\left(R_{3} y(t)\right) t^{\omega-1}\right]\right\} t^{\omega-1} \\
= & \left(R_{1} y(t)\right) t^{\mu-1}+\left(R_{2} y(t)\right) t^{\nu-1}+\left(R_{3} y(t)\right) t^{\omega-1}=Q y(t) .
\end{aligned}
$$

So, $Q$ is a projector operator. From Lemma 3.1, we have $\operatorname{Im} L \subset \operatorname{Ker} Q$. Now, we show the fact that $\operatorname{Ker} Q \subset \operatorname{Im} L$. In fact, for $y \in \operatorname{Ker} Q$, thus $Q y=0$, then we get a system of linear equations with respect to $Q_{1} y, Q_{2} y, Q_{3} y$ as follows:

$$
\left\{\begin{array}{l}
A_{11} Q_{1} y+A_{12} Q_{2} y+A_{13} Q_{3} y=0 \\
A_{21} Q_{1} y+A_{22} Q_{2} y+A_{23} Q_{3} y=0 \\
A_{31} Q_{1} y+A_{32} Q_{2} y+A_{33} Q_{3} y=0 .
\end{array}\right.
$$


Since the determinant of coefficiency for (3.4) is $\Delta^{2}(\mu, v, \omega) \neq 0$, we get that $Q_{1} y=Q_{2} y=$ $Q_{3} y=0$, thus $\operatorname{Ker} Q \subset \operatorname{Im} L$. Therefore, $\operatorname{Ker} Q=\operatorname{Im} L$. For $y \in Y$, set $y=(y-Q y)+Q y$, then $(y-Q y) \in \operatorname{Ker} Q=\operatorname{Im} L, Q y \in \operatorname{Im} Q$. So, $y=\operatorname{Im} L+\operatorname{Im} Q$. Furthermore, for any $y \in \operatorname{Im} L \cap$ $\operatorname{Im} Q$, there exist constants $a, b, c \in \mathbb{R}$ such that $y(t)=a t^{\mu-1}+b t^{\nu-1}+c t^{\omega-1}$ and $Q_{1} y=Q_{2} y=$ $Q_{3} y=0$. Then we also get a system of linear equations with respect to $a, b, c$ as follows:

$$
\left\{\begin{array}{l}
\sum_{i=1}^{l} \alpha_{i}\left(\frac{a}{\mu} \xi_{i}^{\mu}+\frac{b}{v} \xi_{i}^{v}+\frac{c}{\omega} \xi_{i}^{\omega}\right)=0 \\
\sum_{j=1}^{m} \beta_{j}\left[\frac{a}{\mu(\mu+1)} \eta_{j}^{\mu+1}+\frac{b}{v(v+1)} \eta_{j}^{\nu+1}+\frac{c}{\omega(\omega+1)} \eta_{j}^{\omega+1}\right]=0 \\
\frac{2 a}{\mu(\mu+1)(\mu+2)}\left(1-\sum_{k=1}^{n} \gamma_{k} \rho_{k}^{\mu+2}\right)+\frac{2 b}{\nu(v+1)(v+2)}\left(1-\sum_{k=1}^{n} \gamma_{k} \rho_{k}^{v+2}\right) \\
\quad+\frac{2 c}{\omega(\omega+1)(\omega+2)}\left(1-\sum_{k=1}^{n} \gamma_{k} \rho_{k}^{\omega+2}\right)=0
\end{array}\right.
$$

By simple calculation, we obtain that the determinant of coefficiency for (3.5) is

$$
\frac{\Delta(\mu, v, \omega)}{\mu(\mu+1)(\mu+2) v(v+1)(v+2) \omega(\omega+1)(\omega+2)} \neq 0 .
$$

Thus, (3.5) only have zero solutions, that is, $a=b=c=0$. It means that $\operatorname{Im} Q \cap \operatorname{Im} L=\{0\}$. Therefore, $Y=\operatorname{Im} Q \oplus \operatorname{Im} L$. From the above, we get $\operatorname{dim} \operatorname{Ker} L=\operatorname{dim} \operatorname{Im} Q=\operatorname{codim} \operatorname{Im} L=3$. So, $L$ is a Fredholm operator of index zero.

Lemma 3.4 Assume that $\left(\mathrm{H}_{1}\right)$ holds, define the linear operator $K_{p}: \operatorname{Im} L \rightarrow \operatorname{dom} L \cap \operatorname{Ker} P$ by

$$
\left(K_{p} y\right)(t)=\frac{1}{\Gamma(\alpha)} \int_{0}^{t}(t-s)^{\alpha-1} y(s) d s, \quad y \in \operatorname{Im} L,
$$

then $K_{p}$ is the inverse of $\left.L\right|_{\operatorname{dom} L \cap \operatorname{Ker} P}$ and $\left\|K_{p} y\right\|_{X} \leq\|y\|_{1}, \forall y \in \operatorname{Im} L$.

Proof For $y \in \operatorname{Im} L$, then $Q_{1} y=Q_{2} y=Q_{3} y=0$, which is combined with the definition of $K_{p}$ and Lemmas 2.2-2.4, we can check that $K_{p} y \in \operatorname{dom} L \cap \operatorname{Ker} P$. So, $K_{p}$ is well defined on $\operatorname{Im} L$. Obviously, $\left(L K_{p}\right) y(t)=y(t), \forall y \in \operatorname{Im} L$. For $u(t) \in \operatorname{dom} L$, by Lemma 2.2, we have

$$
\left(K_{p} L\right) u(t)=I_{0+}^{\alpha} D_{0+}^{\alpha} u(t)=u(t)+c_{1} t^{\alpha-1}+c_{2} t^{\alpha-2}+c_{3} t^{\alpha-3}+c_{4} t^{\alpha-4}, \quad c_{1}, c_{2}, c_{3}, c_{4} \in \mathbb{R} .
$$

Then $0=\left.I_{0+}^{4-\alpha}\left[\left(K_{p} L\right) u(t)\right]\right|_{t=0}=c_{4}$. It follows from $P\left[\left(K_{p} L\right) u(t)\right]=0$ and $c_{1} t^{\alpha-1}+c_{2} t^{\alpha-2}+$ $c_{3} t^{\alpha-3} \in \operatorname{Ker} L=\operatorname{Im} P$ that $c_{1} t^{\alpha-1}+c_{2} t^{\alpha-2}+c_{3} t^{\alpha-3}=-P u(t)$. Then $\left(K_{p} L\right) u(t)=u(t)-P u(t)$. Therefore, if $u(t) \in \operatorname{dom} L \cap \operatorname{Ker} P$, we have $\left(K_{p} L\right) u(t)=u(t)$. So, $K_{p}$ is the inverse of

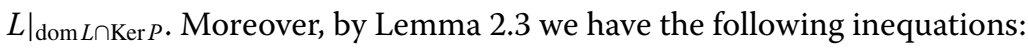

$$
\begin{aligned}
& \left|K_{p} y\right| \leq \frac{1}{\Gamma(\alpha)} \int_{0}^{t}(t-s)^{\alpha-1}|y(s)| d s \leq \frac{1}{\Gamma(\alpha)} \int_{0}^{1}|y(s)| d s \leq\|y\|_{1}, \\
& \left|D_{0+}^{\alpha-3} K_{p} y\right| \leq \frac{1}{2} \int_{0}^{t}(t-s)^{2}|y(s)| d s \leq \frac{1}{2} \int_{0}^{1}|y(s)| d s \leq\|y\|_{1}, \\
& \left|D_{0+}^{\alpha-2} K_{p} y\right| \leq \int_{0}^{t}(t-s)|y(s)| d s \leq \int_{0}^{1}|y(s)| d s=\|y\|_{1}, \\
& \left|D_{0+}^{\alpha-1} K_{p} y\right| \leq \int_{0}^{t}|y(s)| d s \leq \int_{0}^{1}|y(s)| d s=\|y\|_{1} .
\end{aligned}
$$

So, $\left\|K_{p} y\right\|_{X} \leq\|y\|_{1}, \forall y \in \operatorname{Im} L$. 
Lemma 3.5 Assume that $\left(\mathrm{H}_{1}\right)$ holds and $\Omega \subset X$ is an open bounded subset with $\operatorname{dom} L \cap$ $\bar{\Omega} \neq \emptyset$, then $N$ is L-compact on $\bar{\Omega}$.

Proof From $f:[0,1] \times \mathbb{R}^{4} \rightarrow \mathbb{R}$ satisfies the Carathéodory conditions, we can get that $Q N(\bar{\Omega})$ and $(I-Q) N(\bar{\Omega})$ are bounded, that is, there exist constants $\ell^{\prime}, \ell>0$ such that $|Q N u| \leq \ell^{\prime},|(I-Q) N u| \leq \ell, u \in \bar{\Omega}$, a.e. $t \in[0,1]$. So, we only need to show that $K_{p}(I-Q) N$ : $\bar{\Omega} \rightarrow X$ is compact. By Lemma 3.4, $K_{p}(I-Q) N(\bar{\Omega})$ is bounded. It follows from the Lebesgue dominated convergence theorem that $K_{p}(I-Q) N: \bar{\Omega} \rightarrow X$ is continuous. For $0 \leq t_{1}<$ $t_{2} \leq 1, u \in \bar{\Omega}$, we have

$$
\begin{aligned}
\left|K_{p}(I-Q) N u\left(t_{1}\right)-K_{p}(I-Q) N u\left(t_{2}\right)\right| \\
=\frac{1}{\Gamma(\alpha)}\left|\int_{0}^{t_{1}}\left(t_{1}-s\right)^{\alpha-1}(I-Q) N u(s) d s-\int_{0}^{t_{2}}\left(t_{2}-s\right)^{\alpha-1}(I-Q) N u(s) d s\right| \\
\leq \frac{1}{\Gamma(\alpha)}\left|\int_{0}^{t_{1}}\left[\left(t_{1}-s\right)^{\alpha-1}-\left(t_{2}-s\right)^{\alpha-1}\right](I-Q) N u(s) d s\right| \\
\quad+\frac{1}{\Gamma(\alpha)}\left|\int_{t_{1}}^{t_{2}}\left(t_{2}-s\right)^{\alpha-1}(I-Q) N u(s) d s\right| \\
\leq \frac{\ell}{\Gamma(\alpha)} \int_{0}^{t_{1}}\left[\left(t_{2}-s\right)^{\alpha-1}-\left(t_{1}-s\right)^{\alpha-1}\right] d s+\frac{\ell}{\Gamma(\alpha)} \int_{t_{1}}^{t_{2}}\left(t_{2}-s\right)^{\alpha-1} d s \\
=\frac{\ell}{\Gamma(\alpha+1)}\left(t_{2}^{\alpha}-t_{1}^{\alpha}\right) .
\end{aligned}
$$

Since $t^{\alpha}$ is uniformly continuous on $[0,1]$, we get $K_{p}(I-Q) N(\bar{\Omega})$ is equicontinuous. In addition, because the following equations hold:

$$
\begin{aligned}
& D_{0+}^{\alpha-2} K_{p}(I-Q) N u\left(t_{2}\right)-D_{0+}^{\alpha-2} K_{p}(I-Q) N u\left(t_{1}\right)=\int_{t_{1}}^{t_{2}} D_{0+}^{\alpha-1} K_{p}(I-Q) N u(s) d s, \\
& D_{0+}^{\alpha-3} K_{p}(I-Q) N u\left(t_{2}\right)-D_{0+}^{\alpha-3} K_{p}(I-Q) N u\left(t_{1}\right)=\int_{t_{1}}^{t_{2}} D_{0+}^{\alpha-2} K_{p}(I-Q) N u(s) d s,
\end{aligned}
$$

we simply indicate the equicontinuity of $D_{0+}^{\alpha-1} K_{p}(I-Q) N(\bar{\Omega})$. In fact,

$$
\begin{aligned}
& \left|D_{0+}^{\alpha-1} K_{p}(I-Q) N u\left(t_{1}\right)-D_{0+}^{\alpha-1} K_{p}(I-Q) N u\left(t_{2}\right)\right| \\
& \quad=\left|\int_{t_{1}}^{t_{2}}(I-Q) N u(s) d s\right| \leq \ell\left(t_{2}-t_{1}\right) .
\end{aligned}
$$

Since $t$ is uniformly continuous on $[0,1]$, thus $D_{0+}^{\alpha-1} K_{p}(I-Q) N(\bar{\Omega})$ is equicontinuous. By the Arzelà-Ascoli theorem, we obtain that $K_{p}(I-Q) N: \bar{\Omega} \rightarrow X$ is compact.

In order to obtain our main results, we suppose that the following conditions are satisfied:

$\left(\mathrm{H}_{2}\right)$ There exist nonnegative functions $a(t), b(t), c(t), d(t), e(t) \in Y$ such that, for any $\left(u_{1}, u_{2}, u_{3}, u_{4}\right) \in \mathbb{R}^{4}, t \in(0,1)$

$$
\left|f\left(t, u_{1}, u_{2}, u_{3}, u_{4}\right)\right| \leq a(t)\left|u_{1}\right|+b(t)\left|u_{2}\right|+c(t)\left|u_{3}\right|+d(t)\left|u_{4}\right|+e(t)
$$


and

$$
\|a\|_{1}+\|b\|_{1}+\|c\|_{1}+\|d\|_{1}<\frac{1}{(q+1)},
$$

where $q=\max \left\{\frac{1}{\Gamma(\alpha)}+\frac{1}{\Gamma(\alpha-1)}+\frac{1}{\Gamma(\alpha-2)}, \frac{5}{2}\right\}$.

$\left(\mathrm{H}_{3}\right)$ There exists a constant $L>0$ for any $u(t) \in \operatorname{dom} L$, if $\inf _{t \in[0,1]}\left|D_{0+}^{\alpha-1} u(t)\right|>L$, then

$$
D_{0+}^{\alpha-1} u(t) Q_{1} N u(t)>0, \quad \forall t \in[0,1]
$$

or

$$
D_{0+}^{\alpha-1} u(t) Q_{1} N u(t)<0, \quad \forall t \in[0,1]
$$

$\left(\mathrm{H}_{4}\right)$ There exists a constant $M>0$ for any $u(t) \in \operatorname{dom} L$, if $\inf _{t \in[0,1]}\left|D_{0+}^{\alpha-2} u(t)\right|>M$, then

$$
D_{0+}^{\alpha-2} u(t) Q_{2} N u(t)>0, \quad \forall t \in[0,1]
$$

or

$$
D_{0+}^{\alpha-2} u(t) Q_{2} N u(t)<0, \quad \forall t \in[0,1]
$$

$\left(\mathrm{H}_{5}\right)$ There exists a constant $G>0$ for any $u(t) \in \operatorname{dom} L$, if $\inf _{t \in[0,1]}\left|D_{0+}^{\alpha-3} u(t)\right|>G$, then

$$
D_{0+}^{\alpha-3} u(t) Q_{3} N u(t)>0, \quad \forall t \in[0,1]
$$

or

$$
D_{0+}^{\alpha-3} u(t) Q_{3} N u(t)<0, \quad \forall t \in[0,1]
$$

Lemma 3.6 Suppose that $\left(\mathrm{H}_{2}\right)-\left(\mathrm{H}_{5}\right)$ hold, set

$$
\Omega_{1}=\{u \in \operatorname{dom} L \backslash \operatorname{Ker} L: L u=\lambda N u, \lambda \in(0,1)\} .
$$

Then $\Omega_{1}$ is bounded.

Proof For $u \in \Omega_{1}$, we have $N u \in \operatorname{Im} L=\operatorname{Ker} Q$, that is, $Q_{1}(N u(t))=Q_{2}(N u(t))=$ $Q_{3}(N u(t))=0$. Thus, from $\left(\mathrm{H}_{3}\right)-\left(\mathrm{H}_{5}\right)$, we obtain that there exist constants $t_{1}, t_{2}, t_{3} \in[0,1]$ such that $\left|D_{0+}^{\alpha-3} u\left(t_{3}\right)\right| \leq G,\left|D_{0+}^{\alpha-2} u\left(t_{2}\right)\right| \leq M$ and $\left|D_{0+}^{\alpha-1} u\left(t_{1}\right)\right| \leq L$. Since, for all $t \in[0,1]$, the following equations hold:

$$
\begin{aligned}
& D_{0+}^{\alpha-1} u(t)=D_{0+}^{\alpha-1} u\left(t_{1}\right)+\int_{t_{1}}^{t} D_{0+}^{\alpha} u(s) d s, \\
& D_{0+}^{\alpha-2} u(t)=D_{0+}^{\alpha-2} u\left(t_{2}\right)+\int_{t_{2}}^{t} D_{0+}^{\alpha-1} u(s) d s, \\
& D_{0+}^{\alpha-3} u(t)=D_{0+}^{\alpha-3} u\left(t_{3}\right)+\int_{t_{3}}^{t} D_{0+}^{\alpha-2} u(s) d s .
\end{aligned}
$$


Then we have

$$
\begin{aligned}
& \left|D_{0+}^{\alpha-1} u(0)\right| \leq\left\|D_{0+}^{\alpha-1} u(t)\right\|_{\infty} \leq\left|D_{0+}^{\alpha-1} u\left(t_{1}\right)\right|+\left\|D_{0+}^{\alpha} u\right\|_{1} \leq L+\|L u\|_{1} \leq L+\|N u\|_{1}, \\
& \left|D_{0+}^{\alpha-2} u(0)\right| \leq\left\|D_{0+}^{\alpha-2} u(t)\right\|_{\infty} \leq\left|D_{0+}^{\alpha-2} u\left(t_{2}\right)\right|+\left\|D_{0+}^{\alpha-1} u\right\|_{\infty} \leq L+M+\|N u\|_{1},
\end{aligned}
$$

and

$$
\left|D_{0+}^{\alpha-3} u(0)\right| \leq\left\|D_{0+}^{\alpha-3} u(t)\right\|_{\infty} \leq\left|D_{0+}^{\alpha-3} u\left(t_{3}\right)\right|+\left\|D_{0+}^{\alpha-2} u\right\|_{\infty} \leq L+M+G+\|N u\|_{1} \text {. }
$$

By the definition of $P$ and Lemma 2.4, we get

$$
\begin{aligned}
& D_{0+}^{\alpha-3} P u(t)=\frac{1}{2} D_{0+}^{\alpha-1} u(0) t^{2}+D_{0+}^{\alpha-2} u(0) t+D_{0+}^{\alpha-3} u(0), \\
& D_{0+}^{\alpha-2} P u(t)=D_{0+}^{\alpha-1} u(0) t+D_{0+}^{\alpha-2} u(0), \quad D_{0+}^{\alpha-1} P u(t)=D_{0+}^{\alpha-1} u(0) .
\end{aligned}
$$

Thus,

$$
\begin{aligned}
& \left|D_{0+}^{\alpha-3} P u\right| \leq \frac{1}{2}\left|D_{0+}^{\alpha-1} u(0)\right|+\left|D_{0+}^{\alpha-2} u(0)\right|+\left|D_{0+}^{\alpha-3} u(0)\right| \leq \frac{5}{2} L+2 M+G+\frac{5}{2}\|N u\|_{1}, \\
& \left|D_{0+}^{\alpha-2} P u\right| \leq\left|D_{0+}^{\alpha-1} u(0)\right|+\left|D_{0+}^{\alpha-2} u(0)\right| \leq 2 L+M+2\|N u\|_{1}, \\
& \left|D_{0+}^{\alpha-1} P u\right|=\left|D_{0+}^{\alpha-1} u(0)\right| \leq L+\|N u\|_{1} .
\end{aligned}
$$

Because of

$$
\begin{aligned}
|P u| & \leq \frac{1}{\Gamma(\alpha)}\left|D_{0+}^{\alpha-1} u(0)\right|+\frac{1}{\Gamma(\alpha-1)}\left|D_{0+}^{\alpha-2} u(0)\right|+\frac{1}{\Gamma(\alpha-2)}\left|D_{0+}^{\alpha-3} u(0)\right| \\
& \leq \frac{L}{\Gamma(\alpha)}+\frac{L+M}{\Gamma(\alpha-1)}+\frac{L+M+G}{\Gamma(\alpha-2)}+\left(\frac{1}{\Gamma(\alpha)}+\frac{1}{\Gamma(\alpha-1)}+\frac{1}{\Gamma(\alpha-2)}\right)\|N u\|_{1} .
\end{aligned}
$$

Therefore,

$$
\begin{aligned}
\|P u\|_{X} & =\max \left\{\|P u\|_{\infty},\left\|D_{0+}^{\alpha-3} P u\right\|_{\infty},\left\|D_{0+}^{\alpha-2} P u\right\|_{\infty},\left\|D_{0+}^{\alpha-1} P u\right\|_{\infty}\right\} \\
& \leq q L+p M+r G+q\|N u\|_{1},
\end{aligned}
$$

where $p=\max \left\{\frac{1}{\Gamma(\alpha-1)}+\frac{1}{\Gamma(\alpha-2)}, 2\right\}, r=\frac{1}{\Gamma(\alpha-2)}$. Also, for $u \in \Omega_{1}, u \in \operatorname{dom} L \backslash \operatorname{Ker} L$, then $(I-$ $P) u \in \operatorname{dom} L \cap \operatorname{Ker} P, L P u=0$, from Lemma 3.4, we have

$$
\|(I-P) u\|_{X}=\left\|K_{p} L(I-P) u\right\|_{X}=\left\|K_{p} L u\right\|_{X} \leq\|L u\|_{1} \leq\|N u\|_{1} .
$$

It follows from (3.12) and (3.13) that

$$
\begin{aligned}
\|u\|_{X} & =\|P u+(I-P) u\|_{X} \leq\|P u\|_{X}+\|(I-P) u\|_{X} \\
& \leq p M+q L+r G+(q+1)\|N u\|_{1} .
\end{aligned}
$$


By $\left(\mathrm{H}_{2}\right)$, we have

$$
\begin{aligned}
\|N u\|_{1} \leq & \|a\|_{1}\|u\|_{\infty}+\|b\|_{1}\left\|D_{0+}^{\alpha-3} u\right\|_{\infty}+\|c\|_{1}\left\|D_{0+}^{\alpha-2} u\right\|_{\infty} \\
& +\|d\|_{1}\left\|D_{0+}^{\alpha-1} u\right\|_{\infty}+\|e\|_{1} \\
\leq & \left(\|a\|_{1}+\|b\|_{1}+\|c\|_{1}+\|d\|_{1}\right)\left[p M+q L+r G+(q+1)\|N u\|_{1}\right]+\|e\|_{1} .
\end{aligned}
$$

Substituting (3.15) into (3.14), one gets

$$
\|u\|_{X} \leq(q+1) \frac{\left(\|a\|_{1}+\|b\|_{1}+\|c\|_{1}+\|d\|_{1}\right)(p M+q L+r G)+\|e\|_{1}}{1-(q+1)\left(\|a\|_{1}+\|b\|_{1}+\|c\|_{1}+\|d\|_{1}\right)}+p M+q L+r G .
$$

So, $\Omega_{1}$ is bounded.

Lemma 3.7 Suppose that $\left(\mathrm{H}_{3}\right)-\left(\mathrm{H}_{5}\right)$ hold, set

$$
\Omega_{2}=\{u \in \operatorname{Ker} L: N u \in \operatorname{Im} L\}
$$

Then $\Omega_{2}$ is bounded.

Proof For $u \in \Omega_{2}$, then $u \in \operatorname{Ker} L$ and $N u \in \operatorname{Im} L=\operatorname{Ker} Q$, that is, there exist constants $a, b, c \in \mathbb{R}$ such that $u(t)=a t^{\alpha-1}+b t^{\alpha-2}+c t^{\alpha-3}, Q N u(t)=0$. Thus, $Q_{1} N u(t)=Q_{2} N u(t)=$ $Q_{3} N u(t)=0$. By $\left(\mathrm{H}_{3}\right)-\left(\mathrm{H}_{5}\right)$, there exist constants $t_{4}, t_{5}, t_{6} \in[0,1]$ such that $\left|D_{0+}^{\alpha-3} u\left(t_{6}\right)\right| \leq G$, $\left|D_{0+}^{\alpha-2} u\left(t_{5}\right)\right| \leq M$ and $\left|D_{0+}^{\alpha-1} u\left(t_{4}\right)\right| \leq L$, that is,

$$
\begin{aligned}
& \left|D_{0+}^{\alpha-1} u\left(t_{4}\right)\right|=|a \Gamma(\alpha)| \leq L, \quad\left|D_{0+}^{\alpha-2} u\left(t_{5}\right)\right|=\left|a \Gamma(\alpha) t_{5}+b \Gamma(\alpha-1)\right| \leq M, \\
& \left|D_{0+}^{\alpha-3} u\left(t_{6}\right)\right|=\left|a \Gamma(\alpha) t_{6}^{2} / 2+b \Gamma(\alpha-1) t_{6}+c \Gamma(\alpha-2)\right| \leq G .
\end{aligned}
$$

Then

$$
|a| \leq \frac{L}{\Gamma(\alpha)}, \quad|b| \leq \frac{M+L}{\Gamma(\alpha-1)}, \quad|c| \leq \frac{G+M+(3 L / 2)}{\Gamma(\alpha-2)} .
$$

Therefore,

$$
\begin{aligned}
\|u\|_{\infty} \leq|a|+|b|+|c| \leq \frac{L}{\Gamma(\alpha)}+\frac{M+L}{\Gamma(\alpha-1)}+\frac{G+M+(3 L / 2)}{\Gamma(\alpha-2)}, \\
\left\|D_{0+}^{\alpha-1} u\right\|_{\infty} \leq \Gamma(\alpha)|a| \leq L \\
\left\|D_{0+}^{\alpha-2} u\right\|_{\infty} \leq \Gamma(\alpha)|a|+\Gamma(\alpha-1)|b| \leq 2 L+M \\
\left\|D_{0+}^{\alpha-3} u\right\|_{\infty} \leq \frac{1}{2} \Gamma(\alpha)|a|+\Gamma(\alpha-1)|b|+\Gamma(\alpha-2)|c| \\
\leq 3 L+2 M+G .
\end{aligned}
$$

So, $\Omega_{2}$ is bounded.

Lemma 3.8 Suppose that $\left(\mathrm{H}_{3}\right)-\left(\mathrm{H}_{5}\right)$ hold, set

$$
\Omega_{3}=\{u \in \operatorname{Ker} L: \vartheta \lambda J u+(1-\lambda) Q N u=0, \lambda \in[0,1]\} .
$$


Then $\Omega_{3}$ is bounded, where $\vartheta=1$, if (3.6), (3.8), (3.10) hold and $\vartheta=-1$, if (3.7), (3.9), (3.11) hold, $J: \operatorname{Ker} L \rightarrow \operatorname{Im} Q$ is the linear isomorphism defined by

$$
J\left(a t^{\alpha-1}+b t^{\alpha-2}+c t^{\alpha-3}\right)=\frac{1}{\Delta(\mu, \nu, \omega)}\left(a_{1} t^{\mu-1}+b_{1} t^{\nu-1}+c_{1} t^{\omega-1}\right), \quad \forall a, b, c \in \mathbb{R},
$$

where

$$
\begin{aligned}
& a_{1}=\mu(\mu+1)(\mu+2)\left(A_{11} a+A_{12} b+A_{13} c\right), \\
& b_{1}=v(v+1)(v+2)\left(A_{21} a+A_{22} b+A_{23} c\right), \\
& c_{1}=\omega(\omega+1)(\omega+2)\left(A_{31} a+A_{32} b+A_{33} c\right) .
\end{aligned}
$$

Proof Without loss of generality, we suppose that (3.6), (3.8), (3.10) hold, then for any $u \in \Omega_{3}$, there exist constants $a, b, c \in \mathbb{R}$ and $\lambda \in[0,1]$ such that $u(t)=a t^{\alpha-1}+b t^{\alpha-2}+c t^{\alpha-3}$ and $\lambda J u+(1-\lambda) Q N u=0$. Therefore, we get a system of linear equations with respect to $x_{1}, x_{2}, x_{3}$ as follows:

$$
\left\{\begin{array}{l}
A_{11} x_{1}+A_{12} x_{2}+A_{13} x_{3}=0 \\
A_{21} x_{1}+A_{22} x_{2}+A_{23} x_{3}=0 \\
A_{31} x_{1}+A_{32} x_{2}+A_{33} x_{3}=0
\end{array}\right.
$$

where $x_{1}=\lambda a+(1-\lambda) Q_{1} N u(t), x_{2}=\lambda b+(1-\lambda) Q_{2} N u(t), x_{3}=\lambda c+(1-\lambda) Q_{3} N u(t)$. Since the determinant of coefficiency for (3.16) is $\Delta^{2}(\mu, v, \omega) \neq 0$, we get $x_{1}=x_{2}=x_{3}=0$, that is,

$$
\begin{aligned}
& \lambda a+(1-\lambda) Q_{1} N u(t)=0, \\
& \lambda b+(1-\lambda) Q_{2} N u(t)=0, \\
& \lambda c+(1-\lambda) Q_{3} N u(t)=0 .
\end{aligned}
$$

If $\lambda=1$, one has $a=b=c=0$. Obviously, $\Omega_{3}$ is bounded. If $\lambda \in[0,1)$, it follows from (3.6) and (3.17) that we can get $|a| \leq \frac{L}{\Gamma(\alpha)}$; otherwise, by (3.6) and (3.17), a contradiction will be obtained:

$$
0<\lambda a^{2} \Gamma(\alpha)=-(1-\lambda) a \Gamma(\alpha) Q_{1} N u(t)<0 .
$$

Similarly, from (3.8) and (3.18), we have $|b| \leq \frac{M+L}{\Gamma(\alpha-1)}$; if not, by (3.8) and (3.18), we get a contradiction

$$
0<\lambda b^{2} \Gamma(\alpha-1)=-(1-\lambda) b \Gamma(\alpha-1) Q_{2} N u(t)<0 .
$$

From (3.10) and (3.19), we can derive $|c| \leq \frac{M+G+(3 L / 2)}{\Gamma(\alpha-2)}$; else, by (3.10) and (3.19), we obtain a contradiction

$$
0<\lambda c^{2} \Gamma(\alpha-2)=-(1-\lambda) c \Gamma(\alpha-2) Q_{3} N u(t)<0 .
$$

Similarly to the discussion of Lemma $3.7, \Omega_{3}$ is bounded. 
Theorem 3.1 Suppose that $\left(\mathrm{H}_{1}\right)-\left(\mathrm{H}_{5}\right)$ hold. Then problem (1.1) has at least one solution in $X$.

Proof Set $\Omega$ be a bounded open set of $X$ such that $\bigcup_{i=1}^{3} \bar{\Omega}_{i} \subset \Omega$. By Lemma 3.5, $N$ is $L$ compact on $\bar{\Omega}$. From Lemmas 3.6 and 3.7, we get

(i) $L u \neq \lambda N u$ for any $(u, \lambda) \in[(\operatorname{dom} L \backslash \operatorname{Ker} L) \cap \partial \Omega] \times(0,1)$,

(ii) $N u \in \operatorname{Im} L$ for any $u \in \operatorname{Ker} L \cap \partial \Omega$.

In the following, we only need to check that (iii) of Lemma 2.1 is satisfied. Take

$$
H(u, \lambda)=\vartheta \lambda J u+(1-\lambda) Q N u,
$$

where $\vartheta$ is defined as before. According to Lemma 3.8, we derive $H(u, \lambda) \neq 0$ for all $u \in$ $\operatorname{Ker} L \cap \partial \Omega$. Thus, it follows from the homotopy of degree that

$$
\begin{aligned}
\operatorname{deg}\left\{\left.Q N\right|_{\text {Ker } L}, \Omega \cap \operatorname{Ker} L, 0\right\} & =\operatorname{deg}\{H(\cdot, 0), \Omega \cap \operatorname{Ker} L, 0\} \\
& =\operatorname{deg}\{H(\cdot, 1), \Omega \cap \operatorname{Ker} L, 0\} \\
& =\operatorname{deg}\{\vartheta J, \Omega \cap \operatorname{Ker} L, 0\} \neq 0 .
\end{aligned}
$$

Then, by Lemma 2.1, we can get that the operator function $L u=N u$ has at least one solution in $\operatorname{dom} L \cap \bar{\Omega}$, which is equivalent to problem (1.1) that has at least one solution in $X$.

\section{Example}

Example 4.1 Consider the boundary value problems

$$
\left\{\begin{array}{l}
D_{0+}^{3.5} u(t)=f\left(t, u(t), D_{0+}^{0.5} u(t), D_{0+}^{1.5} u(t), D_{0+}^{2.5} u(t)\right), \quad t \in(0,1), \\
\left.I_{0+}^{0.5} u(t)\right|_{t=0}=0, \quad D_{0+}^{2.5} u(0)=\sum_{i=1}^{2} \alpha_{i} D_{0+}^{2.5} u\left(\xi_{i}\right), \\
D_{0+}^{1.5} u(0)=\sum_{j=1}^{2} \beta_{j} D_{0+}^{1.5} u\left(\eta_{j}\right), \quad D_{0+}^{0.5} u(1)=\sum_{k=1}^{3} \gamma_{k} D_{0+}^{0.5} u\left(\rho_{k}\right),
\end{array}\right.
$$

where

$$
\begin{aligned}
& f\left(t, u(t), D_{0+}^{0.5} u(t), D_{0+}^{1.5} u(t), D_{0+}^{2.5} u(t)\right) \\
& \quad=\frac{1}{8} g_{1}(t) D_{0+}^{0.5} u(t)-180 g_{2}(t) h_{1}\left(D_{0+}^{1.5} u(t)\right)+3 h_{2}\left(D_{0+}^{2.5} u(t)\right)+\sin u(t)+\cos t, \\
& g_{1}(t)=\left\{\begin{array}{ll}
0, & 0 \leq t \leq 3 / 4, \\
1, & 3 / 4<t \leq 1,
\end{array} g_{2}(t)= \begin{cases}0, & 0 \leq t \leq 1 / 2, \\
1, & 1 / 2<t \leq 1,\end{cases} \right. \\
& h_{i}(\kappa)= \begin{cases}-1, & \kappa<-1, \\
\kappa, & -1 \leq \kappa \leq 1, \\
1, & \kappa>1 .\end{cases}
\end{aligned}
$$

Take

$$
\begin{aligned}
& \alpha_{1}=-1, \quad \alpha_{2}=2, \quad \xi_{1}=\frac{1}{4}, \quad \xi_{2}=\frac{1}{2}, \\
& \beta_{1}=9, \quad \beta_{2}=-8, \quad \eta_{1}=\frac{2}{3}, \quad \eta_{2}=\frac{3}{4},
\end{aligned}
$$




$$
\begin{array}{ll}
\gamma_{1}=1, & \gamma_{2}=-3, \quad \gamma_{2}=3, \\
\rho_{1}=\frac{1}{4}, & \rho_{2}=\frac{1}{2}, \quad \rho_{3}=\frac{3}{4}, \quad \mu=1, \quad \nu=2, \quad \omega=3,
\end{array}
$$

then

$$
\Delta(1,2,3)=-\frac{575}{2048}, \quad\left|f\left(t, u(t), D_{0+}^{0.5} u(t), D_{0+}^{1.5} u(t), D_{0+}^{2.5} u(t)\right)\right| \leq \frac{1}{8}\left|D_{0+}^{0.5} u(t)\right|+185
$$

Let $a(t)=c(t)=d(t)=0, b(t)=\frac{1}{8}, e(t)=185$, then

$$
\|a\|_{1}+\|b\|_{1}+\|c\|_{1}+\|d\|_{1}=\frac{1}{8}<\frac{2}{7}=\frac{1}{q+1} .
$$

Take $L=1$, note $v(t)=3 h_{2}\left(D_{0_{+}}^{2.5} u(t)\right)+\sin u(t)+\cos t$, if $\left|D_{0+}^{2.5} u(t)\right|>1$, then

$$
D_{0+}^{2.5} u(t) Q_{1} N u(t)=D_{0+}^{2.5} u(t)\left[\int_{1 / 4}^{1 / 2} v(t) d t+\int_{0}^{1 / 2} v(t) d t\right]>0 .
$$

Take $M=1$, since

$$
\left|9 \int_{0}^{2 / 3}(2 / 3-t) v(t) d t-8 \int_{0}^{3 / 4}(3 / 4-t) v(t) d t\right| \leq \frac{85}{4}
$$

if $\left|D_{0+}^{1.5} u(t)\right|>1$, then

$$
\begin{aligned}
D_{0+}^{1.5} u(t) Q_{2} N u(t) & \\
= & D_{0+}^{1.5} u(t)\left[9 \int_{0}^{2 / 3}(2 / 3-t) v(t) d t-8 \int_{0}^{3 / 4}(3 / 4-t) v(t) d t\right. \\
& \left.-1620 \int_{1 / 2}^{2 / 3}(2 / 3-t) h_{1}\left(D_{0+}^{1.5} u(t)\right) d t+1440 \int_{1 / 2}^{3 / 4}(3 / 4-t) h_{1}\left(D_{0+}^{1.5} u(t)\right) d t\right]
\end{aligned}
$$

$>0$.

Take $G=23,040$, because of

$$
\begin{aligned}
& \mid \int_{0}^{1}(1-t)^{2} v(t) d t-\int_{0}^{1 / 4}(1 / 4-t)^{2} v(t) d t+3 \int_{0}^{1 / 2}(1 / 2-t)^{2} v(t) d t \\
& \quad-3 \int_{0}^{3 / 4}(3 / 4-t)^{2} v(t) d t-180 \int_{1 / 2}^{1}(1-t)^{2} h_{1}\left(D_{0+}^{1.5} u(t)\right) d t \\
& \quad+540 \int_{1 / 2}^{3 / 4}(3 / 4-t)^{2} h_{1}\left(D_{0+}^{1.5} u(t)\right) d t \mid \\
& \quad \leq \frac{1415}{96}
\end{aligned}
$$




$$
\begin{aligned}
& \text { if }\left|D_{0+}^{0.5} u(t)\right|>G \text {, then } \\
& \begin{aligned}
D_{0+}^{0.5} u(t) Q_{3} N u(t) \\
=D_{0+}^{0.5} u(t)\left[\int_{0}^{1}(1-t)^{2} v(t) d t-\int_{0}^{1 / 4}(1 / 4-t)^{2} v(t) d t+3 \int_{0}^{1 / 2}(1 / 2-t)^{2} v(t) d t\right. \\
\quad-3 \int_{0}^{3 / 4}(3 / 4-t)^{2} v(t) d t-180 \int_{1 / 2}^{1}(1-t)^{2} h_{1}\left(D_{0+}^{1.5} u(t)\right) d t \\
\left.\quad+540 \int_{1 / 2}^{3 / 4}(3 / 4-t)^{2} h_{1}\left(D_{0+}^{1.5} u(t)\right) d t+\frac{1}{8} \int_{3 / 4}^{1}(1-t)^{2} D_{0+}^{0.5} u(t) d t\right]
\end{aligned}
\end{aligned}
$$

$>0$.

By Theorem 3.1, boundary value problems (4.1) have at least one solution.

\section{Conclusion}

In this paper, some sufficient conditions are established for the existence of solutions for a class of fractional multi-point boundary value problems at resonance with threedimensional kernel. The main result of this paper is obtained by using Mawhin's continuation theorem. Compared with previous work, the main difficulties in this paper are as follows. First, as we know, by the way of Mawhin's continuation theorem, the higher the dimension of kernel is, the more difficult it is to construct the projections $P$ and $Q$. Second, the main difficulty lies in evaluating prior bounds. Third, it is difficult to construct an example.

\section{Acknowledgements}

This research is supported by the National Natural Science Foundation of China (No. 11271364).

\section{Competing interests}

The authors declare that they have no competing interests.

\section{Authors' contributions}

The authors have made equal contributions to each part of this paper. All the authors read and approved the final manuscript.

\section{Publisher's Note}

Springer Nature remains neutral with regard to jurisdictional claims in published maps and institutional affiliations.

Received: 22 September 2017 Accepted: 28 December 2017 Published online: 15 January 2018

\section{References}

1. Podlubny, I: Geometric and physical interpretation of fractional integration and fractional differentiation. Fract. Calc. Appl. Anal. 5(4), 367-386 (2002). Dedicated to the 60th anniversary of Prof. Francesco Mainardi

2. Miller, KS, Ross, B: An Introduction to the Fractional Calculus and Fractional Differential Equations. Wiley, New York (1993)

3. Kilbas, AA, Srivastava, HM, Trujillo, J): Theory and Applications of Fractional Differential Equations. Elsevier, Amsterdam (2006)

4. Zhou, Y, Wang, J, Zhang, L: Basic Theory of Fractional Differential Equations. World Scientific, Singapore (2016)

5. Podlubny, I: Fractional Differential Equations: An Introduction to Fractional Derivatives, Fractional Differential Equations, to Methods of Their Solution and Some of Their Applications. Academic Press, San Diego (1998)

6. Magin, RL: Fractional calculus models of complex dynamics in biological tissues. Comput. Math. Appl. 59(5), 1586-1593 (2010)

7. El-Saka, HA: The fractional-order SIS epidemic model with variable population size. J. Egypt. Math. Soc. 22(1), 50-54 (2014)

8. Shabibi, M, Postolache, M, Rezapour, S, Vaezpour, SM: Investigation of a multi-singular pointwise defined fractional integro-differential equation. J. Math. Anal. 7(5), 61-77 (2016)

9. Shabibi, M, Rezapour, Sh, Vaezpour, S: A singular fractional integro-differential equation. UPB Sci. Bull., Ser. A, Appl. Math. Phys. 79(1), 109-118 (2017) 
10. Khalil, H, Khan, RA, Baleanu, D, Rashidi, MM: Some new operational matrices and its application to fractional order Poisson equations with integral type boundary constrains. Comput. Appl. Math. (2016). https://doi.org/10.1016/j.camwa.2016.04.014

11. Baleanu, D, Uğurlu, E: Regular fractional dissipative boundary value problems. Adv. Differ. Equ. 2016, Article ID 175 (2016)

12. Jiao, F, Zhou, Y: Existence of solutions for a class of fractional boundary value problems via critical point theory. Comput. Math. Appl. 62(3), 1181-1199 (2011)

13. Ge, B, Rădulescu, V, Zhang, J: Infinitely many positive solutions of fractional boundary value problems. Topol. Methods Nonlinear Anal. 49(2), 647-664 (2017)

14. Boucenna, A, Moussaoui, T: Existence results for a fractional boundary value problem via critical point theory. Acta Univ. Palacki. Olomuc., Fac. Rerum Nat., Math. 54(1), 47-64 (2015)

15. Rezapour, Sh, Shabibi, M: A singular fractional differential equation with Riemann-Liouville integral boundary condition. J. Adv. Math. Stud. 8(1), 80-88 (2015)

16. Baleanu, D, Hedayati, V, Rezapour, S: On two fractional differential inclusions. SpringerPlus 5, Article ID 882 (2016)

17. Agarwal, RP, O'Regan, D, Staněk, S: Positive solutions for Dirichlet problems of singular nonlinear fractional differential equations. J. Math. Anal. Appl. 371(1), 57-68 (2010)

18. Bai, Z, Lü, H: Positive solutions for boundary value problem of nonlinear fractional differential equation. J. Math. Anal. Appl. 311(2), 495-505 (2005)

19. Liu, X, Jia, M, Ge, W: The method of lower and upper solutions for mixed fractional four-point boundary value problem with p-Laplacian operator. Appl. Math. Lett. 65, 56-62 (2017)

20. Jia, M, Liu, X: Multiplicity of solutions for integral boundary value problems of fractional differential equations with upper and lower solutions. Appl. Math. Comput. 232, 313-323 (2014)

21. Zhang, W, Liu, W, Chen, T: Solvability for a fractional $p$-Laplacian multipoint boundary value problem at resonance on infinite interval. Adv. Differ. Equ. 2016, Article ID 183 (2016)

22. Chen, $Y$, Tang, $X$ : Solvability of sequential fractional order multi-point boundary value problems at resonance. Appl. Math. Comput. 218(14), 7638-7648 (2012)

23. Bai, Z, Zhang, Y: The existence of solutions for a fractional multi-point boundary value problem. Comput. Math. Appl. 60(8), 2364-2372 (2010)

24. Jiang, W: The existence of solutions to boundary value problems of fractional differential equations at resonance. Nonlinear Anal. 74(5), 1987-1994 (2011)

25. Jiang, W: Solvability of fractional differential equations with $p$-Laplacian at resonance. Appl. Math. Comput. 260 48-56 (2015)

26. Baleanu, D, Mousalou, A, Rezapour, S: A new method for investigating approximate solutions of some fractional integro-differential equations involving the Caputo-Fabrizio derivative. Adv. Differ. Equ. 2017, Article ID 51 (2017)

27. Baleanu, D, Mousalou, A, Rezapour, S: On the existence of solutions for some infinite coefficient-symmetric Caputo-Fabrizio fractional integro-differential equations. Bound. Value Probl. 2017, Article ID 145 (2017)

28. Aydogan, S, Baleanu, D, Mousalou, A, Rezapour, S: On approximate solutions for two higher-order Caputo-Fabrizio fractional integro-differential equations. Adv. Differ. Equ. 2017, Article ID 221 (2017)

29. De La Sen, M, Hedayati, V, Gholizade Atani, Y, Rezapour, S: The existence and numerical solution for a k-dimensiona system of multi-term fractional integro-differential equations. Nonlinear Anal., Model. Control 22(2), 188-209 (2017)

30. Khalil, H, Khan, R, Baleanu, D, Saker, S: Approximate solution of linear and nonlinear fractional differential equations under m-point local and nonlocal boundary conditions. Adv. Differ. Equ. 2016, Article ID 177 (2016)

31. Mawhin, J: Topological Degree and Boundary Value Problems for Nonlinear Differential Equations: Topological Methods for Ordinary Differential Equations. Springer, Berlin (1993)

32. Mawhin, J: Topological Degree Methods in Nonlinear Boundary Value Problems. NSF-CBMS Regional Conference Series in Mathematics, vol. 40. Am. Math. Soc., Providence (1979)

33. Lin, X, Zhao, B, Du, Z: A third-order multi-point boundary value problem at resonance with one three dimensional kernel space. Carpath. J. Math. 30(1), 93-100 (2014)

34. Li, S, Yin, J, Du, Z: Solutions to third-order multi-point boundary-value problems at resonance with three dimensional kernels. Electron. J. Differ. Equ. 2014, Article ID 61 (2014)

35. Liang, J, Ren, L, Zhao, Z: Existence of solutions for a multi-point boundary value problems with three dimension kernel at resonance. Q. J. Math. 26(1), 138-143 (2011)

36. Zhao, Z, Liang, J, Ren, L: A multi-point boundary value problems with three dimension kernel at resonance. Adv. Math. 38(3), 345-358 (2009)

\section{Submit your manuscript to a SpringerOpen ${ }^{\circ}$ journal and benefit from:}

- Convenient online submission

- Rigorous peer review

- Open access: articles freely available online

- High visibility within the field

Retaining the copyright to your article

Submit your next manuscript at $>$ springeropen.com 